\title{
Tinnitus and Patterns of Hearing Loss
}

\author{
Christine M. Tan, ${ }^{1}$ Wendy Lecluyse, ${ }^{3}$ Don McFerran, ${ }^{2}$ and Ray Meddis ${ }^{1}$ \\ ${ }^{1}$ Department of Psychology, University of Essex, Colchester, CO4 3SO, UK \\ ${ }^{2}$ ENT Department, Essex County Hospital, Colchester, CO3 3NB, UK \\ ${ }^{3}$ School of Applied Social Sciences, University Campus Suffolk, Ipswich, IP4 1OJ, UK
}

Received: 25 October 2011; Accepted: 3 January 2013; Online publication: 18 January 2013

\begin{abstract}
Tinnitus is strongly linked with the presence of damaged hearing. However, it is not known why tinnitus afflicts only some, and not all, hearingimpaired listeners. One possibility is that tinnitus patients have specific inner ear damage that triggers tinnitus. In this study, differences in cochlear function inferred from psychophysical measures were measured between hearing-impaired listeners with tinnitus and hearing-impaired listeners without tinnitus. Despite having similar average hearing loss, tinnitus patients were observed to have better frequency selectivity and compression than those without tinnitus. The results suggest that the presence of subjective tinnitus may not be strongly associated to outer hair cell impairment, at least where hearing impairment is evident. The results also show a different average pattern of hearing impairment amongst the tinnitus patients, consistent with the suggestion that inner hair cell dysfunction with subsequent reduced auditory innervation is a possible trigger of tinnitus.
\end{abstract}

Keywords: tinnitus, hearing loss, psychoacoustics, absolute thresholds, frequency selectivity, compression

\section{INTRODUCTION}

Tinnitus is commonly defined as the perception of sounds in the absence of an external source. This sometimes debilitating condition is prevalent in both the aging population (Lockwood et al. 2002) and

Correspondence to: Ray Meddis · Department of Psychology · University of Essex·Colchester, CO4 3SQ, UK. rmeddis@essex.ac.uk amongst the hearing-impaired generally (Davis and El Refaie 2000). The strong link between the presence of tinnitus and damaged hearing supports the hypothesis that both may have a common cause probably in the auditory periphery. However, the perception of tinnitus is reported to persist even after cochlear ablation or auditory nerve section (Sasaki et al. 1981). This suggests that tinnitus is a central phenomenon, and plastic changes in central auditory structures are currently thought to generate and maintain the perception of tinnitus (Dehmel et al. 2012; Kaltenbach et al. 2005). It is, however, possible that these plastic changes are initiated by peripheral dysfunction although it is not certain what specific damage in the periphery might trigger them.

It is not known, for example, why some people with a hearing impairment develop tinnitus while others do not. If tinnitus does indeed have an origin in dysfunction of the auditory periphery, it might be hypothesised that tinnitus patients comprise a subgroup of hearing impairment. This hypothesis has never been explored by a detailed assessment of the hearing of tinnitus patients in comparison with a control group of equally impaired individuals who do not report tinnitus. This study set out to make this assessment using measurements that were specifically chosen to explore the extent to which outer hair cell $(\mathrm{OHC})$ dysfunction might be involved.

Sensorineural hearing impairment may involve either outer or inner hair cell systems (or both) to variable degrees (Nordmann et al. 2000). OHCs were believed to be more vulnerable to damage than inner hair cells (IHCs) (Hawkins 1973), but recently, IHC dysfunction with subsequent neural degeneration has been shown to co-exist with functional OHCs (Kujawa and Liberman 2009). It is unclear which specific type of inner ear damage might trigger the central changes and resulting perception of tinnitus although a robust relationship was found between tinnitus and the 
absence of afferent fibres to central structures (Bauer et al. 2007). This might suggest that OHC dysfunction is not central to the perception of tinnitus. Other forms of peripheral dysfunction may be involved and have a more prevalent influence on those hearingimpaired listeners who perceive tinnitus. It was decided to measure frequency selectivity and compression as well as absolute thresholds because all of these have been proposed to reflect OHC behaviour (Evans 1975; Liberman and Dodds 1984; Hicks and Bacon 1999; Nelson et al. 2001; Robles and Ruggero 2001; Lopez-Poveda et al. 2005).

This study tests the hypothesis that differences in cochlear function exist between hearing-impaired listeners with tinnitus and hearing-impaired listeners without tinnitus. Psychophysical assessments were made of (1) absolute thresholds, (2) compression and (3) frequency selectivity on the assumption that OHC dysfunction would be reflected in both reduced compression and reduced frequency selectivity. The psychophysical methods that are commonly used in the laboratory for these purposes are time-consuming and it was therefore decided to use more recent techniques that have been optimised for efficient data collection with untrained observers. These methods have previously been evaluated using subjects with a wide range of ages, with normal and impaired hearing (Meddis et al. 2010) and were used without further adaptation. The measurements were made on two groups with impaired hearing: one with tinnitus and one without. A reference group of younger persons with good hearing was also included to compensate for the absence of published norms for the compression and frequency selectivity measurements.

\section{METHODS}

\section{Participants}

Two groups of impaired listeners were studied, 27 with tinnitus (mean age 59, stdev $=10$ years) and 15 without tinnitus (mean age 64, stdev $=15$ years). These were volunteers who responded to an article in a university newsletter or who were told of the project by local healthcare professionals. An additional reference group of 19 younger listeners with good hearing and without tinnitus (mean age 32 , stdev $=9$ years) was recruited from university staff, students and visitors to the laboratory. The purpose of this additional control group was to establish a good-hearing baseline. Screening was performed to exclude suspected outer/middle ear abnormalities. Somatosensory and other conditions that are typically associated with tinnitus (temporomandibular joint disorders, vestibular schwannoma, Ménière's disease) were also excluded from the study. Strict criteria were used to define the presence of tinnitus. Tinnitus had to be permanently perceived in all acoustic environments (not only in quiet surroundings). Participants who reported temporary tinnitus with a sudden onset that lasted only a few seconds were not included in the study. All tinnitus participants completed the Tinnitus Handicap Inventory (THI), a questionnaire that evaluates the impact of the severity of tinnitus on an individual (Newman et al. 1996). Informed consent was obtained from all participants in accordance with the procedures approved by the Essex Research Ethics Committee $(08 / \mathrm{H} 0301 / 134)$ and by the ethics committee at the University of Essex.

\section{Procedure}

Target and masker signals were all sinusoids that were digitally generated at a sampling rate of $96,000 \mathrm{~Hz}$ and ramped with cosine onset and offset times of 0.004 s. Thresholds were measured using a cued single-interval procedure (Lecluyse and Meddis 2009). For all three measures, the aim was to determine the $50 \%$ detection point for a target tone using an adaptive one-up/one-down tracking procedure. Absolute thresholds were measured by adjusting the level of the target tone. For compression and frequency selectivity tests, thresholds were measured using fixed-level target tones $(10 \mathrm{~dB}$ SL) while a preceding masker was adaptively adjusted.

All test stimuli were preceded by a cue stimulus that was identical in all respects to the test stimulus, except for a small difference in the level of either the target or the masker (see below) that rendered the target component easier to hear. This cue stimulus served to prepare the listener for the test stimulus and reminded him/her of the frequencies being used, and how the target sounded when it followed the masker. The presence of the cue stimulus should not be confused with a two-alternative forced-choice procedure; its function was simply to reduce confusion and the need for extensive training. In practice, listeners were asked to identify how many target tones were heard ('none', 'one' or 'two'). When the listener reported two targets, it was assumed that both the cue target and the test target had been heard. If only one target was reported, it was assumed that only the cue target had been heard and the more difficult test target had not been heard. The cue and the test signals were always separated by 0.5 s. On $20 \%$ of trials, the target tone was omitted from the test stimulus as a check. False positives resulted in immediate feedback and the run was restarted. False positives were rare except at the very beginning of testing during a small number of orienting runs. No other feedback was given. Statistical analysis was performed using the analysis of variance general 
linear model allowing for missing data (IBM SPSS statistics package) with target frequency and tinnitus conditions as fixed variables.

The sinusoidal signals were generated by an MAudio Audiophile 2496, 24-bit sound card, which ran on a Windows XP operating system. The stimuli were presented monaurally through a Sennheiser HD600 circumaural headphone. No tones were presented at levels greater than $100 \mathrm{~dB}$ SPL. When the adaptive procedure moved above this level, the run was abandoned and treated as missing data. Participants were tested individually in a double-walled, soundattenuated room. Participants registered their decisions on a Cedrus RB-34 response pad.

\section{Absolute thresholds}

These were measured with $0.25 \mathrm{~s}$ sinusoidal tones, at signal frequencies of $250,500,1,000,2,000,4,000$ and $6,000 \mathrm{~Hz}$. The measurements were averaged across three runs. Between trials, the test-tone level was adjusted in steps of $2 \mathrm{~dB}$. During these trials, the cue stimulus was the same tone as the test tone but presented at a 10-dB higher level and, therefore, almost always above threshold.

\section{Compression}

Compression measurements were made using a forward-masking paradigm, and expressed as temporal masking curves (TMCs) (Nelson et al. 2001; LopezPoveda et al. 2003). These were measured at target frequencies of 250,500, 1,000, 2,000, 4,000 and $6,000 \mathrm{~Hz}$ and averaged across three runs. In this task, the target tone was a fixed-level, $0.016 \mathrm{~s}$ tone presented at $10 \mathrm{~dB}$ SL. It was preceded by a 0.108 -s masking tone whose level was adaptively varied in 2-dB steps. The frequency of the masker was always the same as that of the target. The masker-target gap was randomly presented at 0.02 , $0.04,0.05,0.06$ and $0.08 \mathrm{~s}$.

Participants heard the following sequence: 'beepclick, beep-click' where the first 'beep-click' is the cue stimulus consisting of the cue-masker followed by the cue-target, while the second 'beep-click' is the test stimulus consisting of the test-masker followed by the test-target. Participants were informed that the gap between the 'beep' (masker signal) and the 'click' (target signal) would change between runs. They were instructed to count only the number of 'clicks' (target signals) heard and to ignore the interfering 'beeps' (masker signal). It was anticipated that the level of the (variable) masker required to mask the target would increase as the masker-target gap increased. The cue stimulus was the same as the test stimulus except for the level of the cue-masker, which was always $10 \mathrm{~dB}$ weaker than the test-masker, making the cue-target more audible.

\section{Frequency selectivity}

These measurements were made using a forwardmasking paradigm and expressed as iso-forward masking contours (IFMCs) (Meddis et al. 2010). Measurements were made using target frequencies of $250,500,1,000,2,000,4,000$ and $6,000 \mathrm{~Hz}$ and averaged across three runs. In this task, the target tone was a fixed-level, $0.016 \mathrm{~s}$ tone presented at $10 \mathrm{~dB}$ SL. It was preceded by a 0.108 -s masking tone whose level was adaptively varied in 2-dB steps. The maskertarget gap was fixed at $0.01 \mathrm{~s}$. The masker frequencies were randomly varied between runs and presented at masker/target frequency ratios of 0.5, 0.7, 0.9, 1, 1.1, 1.3 and 1.6.

Participants heard a similar sequence of sounds to that described for the compression measurements. Participants were instructed to count only the number of 'clicks' (target signal) heard and to ignore the interfering 'beeps' (masker signal). It was anticipated that the level of the (variable) masker required to mask the target would increase as the masker-target frequency difference increased. The cue stimulus was the same as the test stimulus except for the level of the cue-masker, which was always $10 \mathrm{~dB}$ weaker than the test-masker, making the cue-target more audible.

The probe levels (10 dB SL) were necessarily higher for hearing-impaired subjects. To assess the effect of higher probe levels, a subgroup of four of the participants with good hearing was reassessed using four probe levels $(29,39,49$ and $59 \mathrm{~dB}$ SPL) at three probe frequencies $(250,1,000$ and $6,000 \mathrm{~Hz})$.

\section{Data analysis}

Group absolute threshold averages were calculated by averaging across all frequencies measured. When individual data points were missing because the required level of the tone or masker was too high, the average was treated as missing data. Compression was given a numerical value for comparison purposes by estimating the slope of the TMC function. The slope was quantified as the rise in masker threshold per 100-ms increase in masker-target gap. Frequency selectivity was quantified by estimating the 'depth' of the IFMC in terms of the difference between the masker threshold at the expected tip of the function (masker frequency=target frequency) with the mean masker threshold at two points in the tail corresponding to masker frequencies of 0.7 and 1.3 times the target frequency. Efforts to estimate tuning more conventionally using a $Q_{10}$ value were frustrated 
because some impaired functions were virtually flat or occasionally slightly inverted.

\section{RESULTS}

The mean THI score for the tinnitus participants was 17.1 (stdev 7.6; range 6-28) indicating mainly mild, well-tolerated tinnitus. Absolute thresholds across frequency for the good-hearing and no-tinnitus groups are illustrated in Figure $1 \mathrm{~A}$ and $\mathrm{B}$, while the thresholds for the tinnitus group are given in Figure 1C. The mean absolute threshold (across frequencies) for the impaired no-tinnitus group was $41 \mathrm{~dB}$ SPL, and for the tinnitus group, it was $34 \mathrm{~dB}$ SPL. Many of the individual thresholds in the tinnitus group were within the normal range while this happened only occasionally in the no-tinnitus group.

To facilitate comparisons between the two groups equated for mean (across-frequency) threshold, participants in the tinnitus group were successively removed in order of threshold level until the average threshold for those remaining was $42 \mathrm{~dB}$ and therefore comparable to the no-tinnitus average. This is called the 'tinnitus reduced sample' and is illustrated in Figure 1D and contains 15 remaining participants. The mean age in the tinnitus reduced sample was 62 years and matched closely to the no-tinnitus sample (64 years). This means that the tinnitus reduced sample and the no-tinnitus sample were matched for mean (across-frequency) threshold and for age. It is noticeable that the pattern of absolute thresholds across frequency for the non-tinnitus group showed considerable heterogeneity and this may reflect a variety of different pathologies. On the other hand, the reduced tinnitus sample showed a large number of threshold profiles characterised by relatively good low-frequency hearing and a highfrequency loss.

The mean THI score for those in the reduced tinnitus sample is 15.6 (stdev 7.7; range, 6-26), while the corresponding mean for those removed is 18.9 (stdev 7.5). In other words, the participants with the lower thresholds unexpectedly reported stronger tinnitus although the difference was not statistically significant.

The auditory profiles for the good-hearing reference sample, no-tinnitus sample, tinnitus sample and tinnitus reduced sample are shown in Figure 2A-D. The TMC indicator of compression is shown at the top of each panel where it can be seen that the slopes are considerably steeper for the good-hearing reference group than for either of the impaired groups. The inset numbers are the slope of the line expressed as decibel rise per $100 \mathrm{~ms}$. The second row shows average absolute thresholds (blue line) and average IFMCs (red lines) for each target frequency. The average IFMC frequency selectivity functions (tuning curves) showed sharp tuning for the good-hearing group that was not replicated for either of the impaired groups. The unfilled red circles indicate the target frequency. The numerical indicator of

\section{A good hearing}

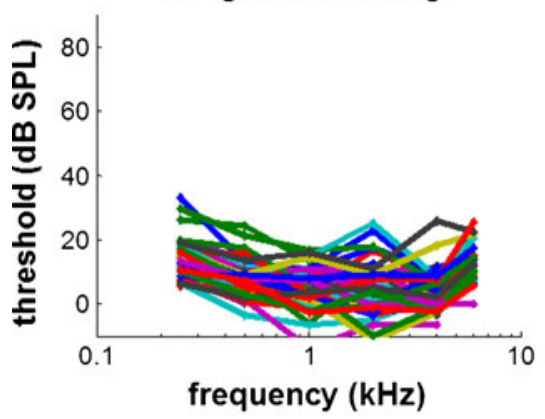

C tinnitus (all)

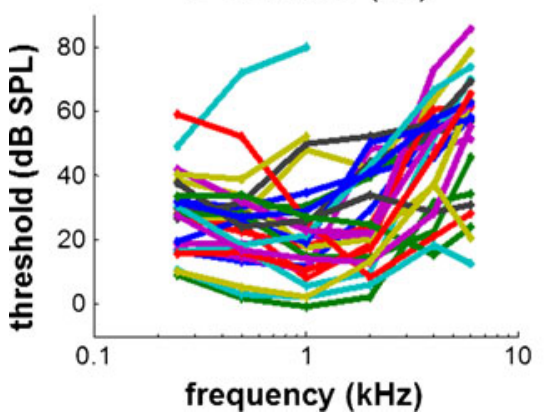

B no tinnitus

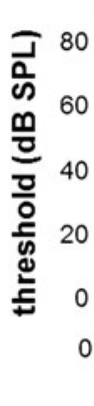

FIG. 1. Absolute thresholds across frequencies for four samples of participants: A good-hearing; B impaired, no tinnitus; C all impaired with tinnitus; and D matched-threshold tinnitus reduced sample (see text).

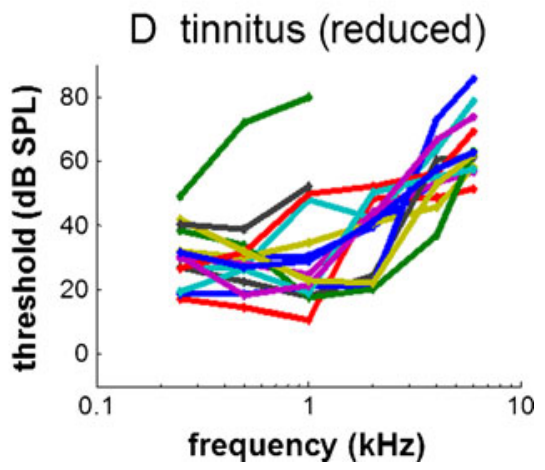




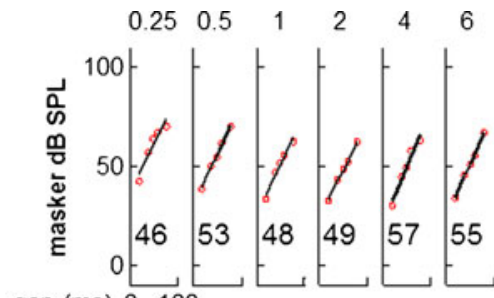

gap (ms) $0 \quad 100$

A good hearing
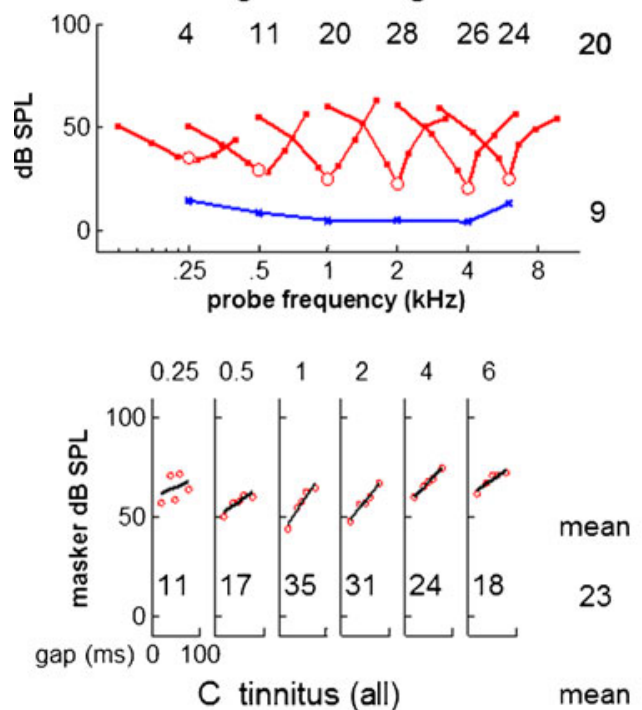

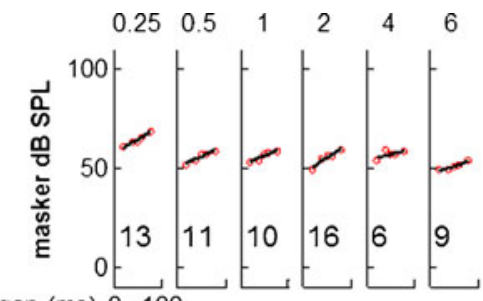

gap (ms) $0 \quad 100$

B no tinnitus
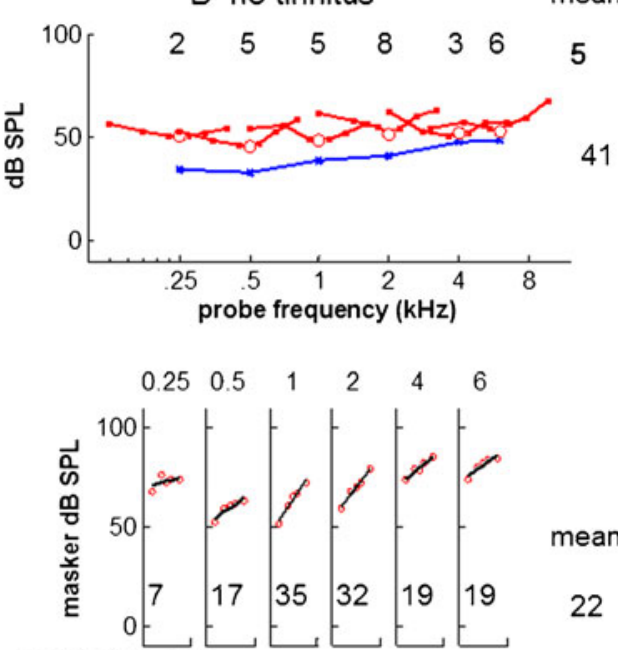

gap (ms) $0 \quad 100$
FIG. 2. Mean auditory profiles for four samples of participants: A good-hearing; B impaired, no-tinnitus; $\mathbf{C}$ all impaired with tinnitus; and D high-threshold tinnitus reduced sample. Within each panel, the top row shows TMCs (reflecting compression, see text) for each target frequency. Individual unfilled red circles are the average across all participants in this group. The black line is the best-fit straight line to these data. The inset values are their estimated slopes. The bottom half of each panel shows the average IFMCs for the group (red lines). The inset values indicate the 'depth' of the IFMC (see text). The lowest line connects the absolute thresholds (decibel sound pressure level). Inset values to the right of the panel are the mean values across frequency.
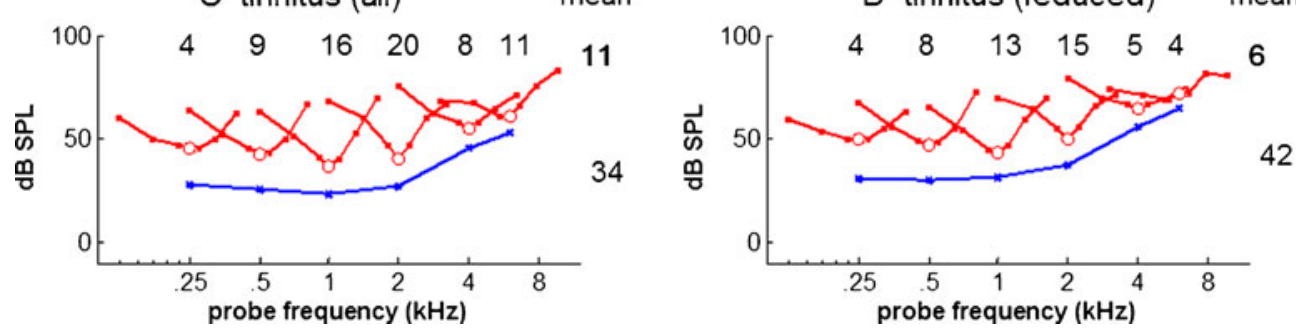

average depth for the IFMCs is given above each tuning curve. Mean values across all target frequencies is given at the end of the rows.

A quantitative analysis for all three measures is given in Figure 3 for the no-tinnitus group, reduced tinnitus group and good-hearing reference group.
Absolute thresholds are compared in Figure 3A showing systematic differences that can be seen between the two tinnitus samples at different frequencies. The average (across-frequency) threshold is now approximately the same for the no-tinnitus and reduced tinnitus groups, and none of the individual

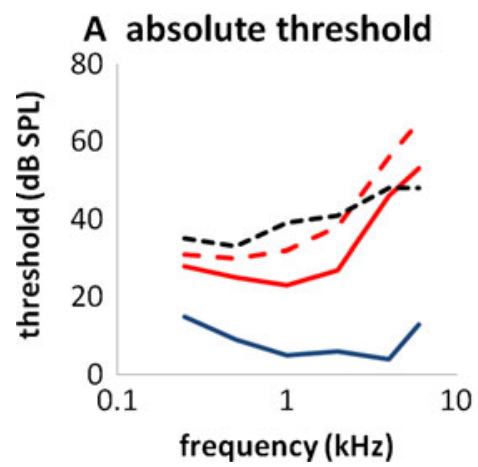

FIG. 3. Comparisons between samples of $\mathbf{A}$ absolute thresholds, B TMC slopes and C IFMC depth. The values in the charts are based on the inset values shown in Figure 2. The dashed lines are from the tinnitus reduced (higher thresholds) sample. The filled squares in C

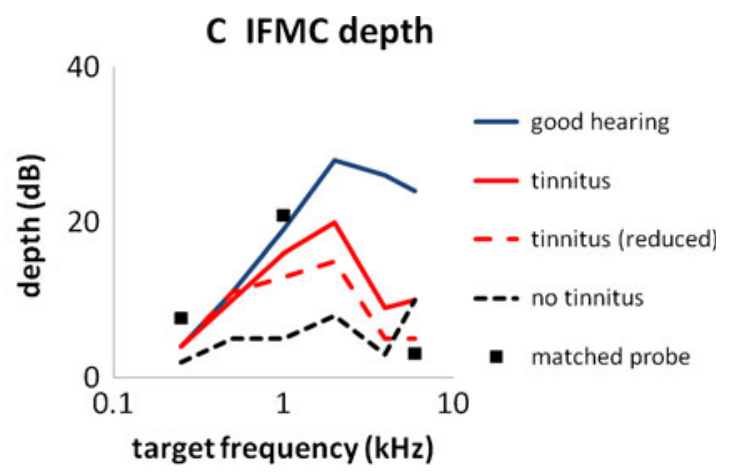

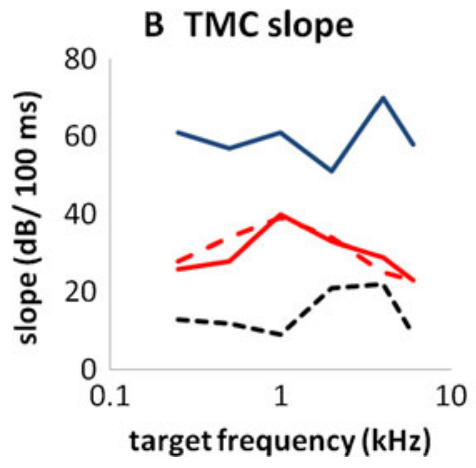

are the IFMC depths for the good-hearing sample when tested using high-probe levels $(39,49$ and $59 \mathrm{~dB}$ SPL) chosen to be close to the average levels used with the tinnitus group (see Fig. 4A-C). 
differences at different target frequencies are statistically significantly different. Nevertheless, there was a tendency for the tinnitus group to have lower thresholds at lower frequencies but higher thresholds at 4 and $6 \mathrm{kHz}$. The TMC slopes also show consistent differences across all frequencies except at $250 \mathrm{~Hz}$. This is statistically significant $\left(F_{(1,5)}=16.1, P<0.001\right)$. The slopes in the tinnitus groups were on average twice as great as those for the no-tinnitus group suggesting more residual compression (Nelson et al. 2001; Lopez-Poveda et al. 2005). The pattern of IFMC depth measurements is more complicated. At middle frequencies $(500,1,000$ and 2,000 $\mathrm{Hz})$, the depth was greater for the tinnitus group indicating greater frequency selectivity. No difference could be seen at $250 \mathrm{~Hz}$ where all groups (including good-hearing) show very wide tuning curves. The difference between the tinnitus conditions was statistically significant $\left(F_{(1}\right.$, 134) $=9.03, P=0.003$ ), but there was also an almost significant frequency $\times$ tinnitus condition interaction $\left(F_{(5,134)}=2.6, P=0.027\right)$.

The irregular pattern of the frequency selectivity results was explored further by testing four listeners with good hearing at four probe levels $(29,39,49$ and $59 \mathrm{~dB}$ SPL) and three probe frequencies (250, 1,000 and $6,000 \mathrm{~Hz}$ ). The results averaged across listeners are shown in Figure 4. Probes at $250 \mathrm{~Hz}$ showed no little or no change in depth as the probe level is increased. However, 1- and 6-kHz probes showed systematic reductions in depth estimates at higher probe levels, with the greatest effect at $6 \mathrm{kHz}$ as shown in Figure 4D. The scale of these reductions was broadly consistent with the differences between the good-hearing sample and the tinnitus sample, if the differences in absolute threshold (and, it follows, probe level) were taken into account. Mean IFMC depth results for the good-hearing group at 250, 1,000 and $6,000 \mathrm{~Hz}$ were found for probe levels 39, 49 and $59 \mathrm{~dB}$ SPL. These probe levels were the same as the average probe levels used to test the hearing-impaired listeners. The mean IFMC depth values were superimposed as black squares on the data in Figure 3C to show the similarity between the goodhearing and tinnitus samples.

\section{DISCUSSION}

The good-hearing reference group showed the expected patterns of (1) low absolute thresholds, (2) steep TMCs, indicating good compression and (3) deep IFMCs, indicating good frequency selectivity. Both impaired groups had worse indicators on all three measures. Some differences were, however, also observed between the tinnitus and no-tinnitus group. The complete tinnitus sample was characterised by lower overall thresholds, greater residual compression and better tuning than the no-tinnitus group in the mid-frequency range. The pattern of absolute thresholds across frequency in the tinnitus groups was mainly that of a high-frequency loss while the no-tinnitus groups showed a wider range of patterns. This is not altogether surprising since the no-tinnitus group is defined in terms of the absence of a feature and may well contain a range of different pathologies. The relative homogeneity of the tinnitus group in this respect suggests that they may have a common pathology. Whether or not this is
A $250 \mathrm{~Hz}$

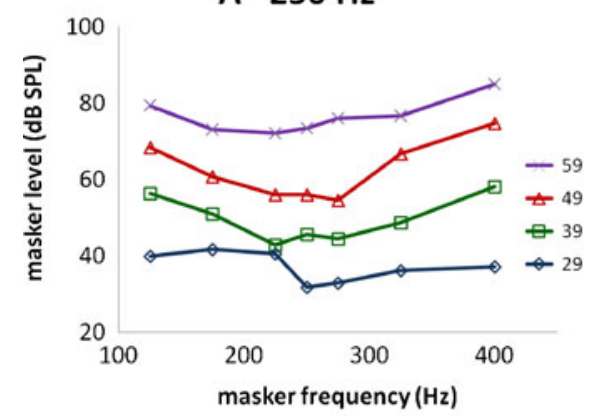

C $6 \mathrm{kHz}$

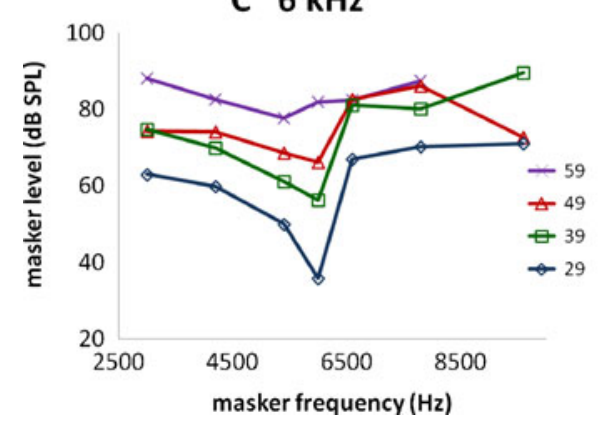

B $1 \mathrm{kHz}$

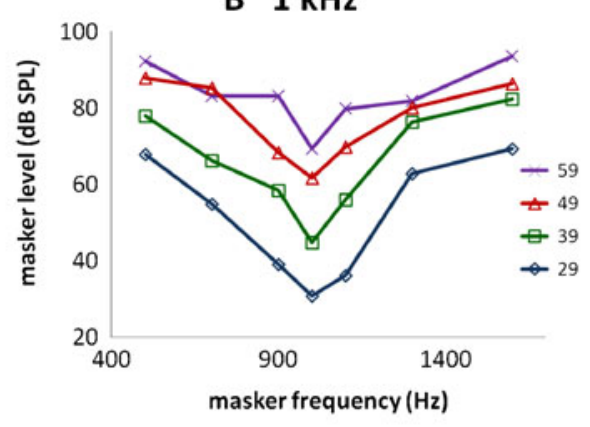

D IFMC depth

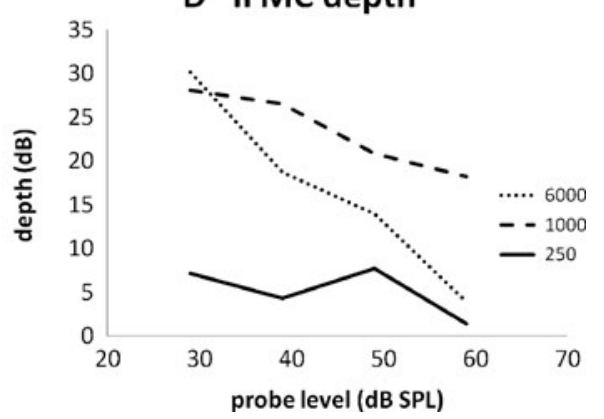

FIG. 4. IFMCs measured in good-hearing participants, at four probe levels and at three probe frequencies: A $250 \mathrm{~Hz}$, B 1,000 Hz and C 6,000 Hz. D Summary of the depth assessment for each IFMC. 
true, the data lead to the expectation that a patient presenting with a tinnitus complaint is likely also to have a high-frequency loss.

Absolute thresholds were lower for the full tinnitus sample. This might reflect the fact that they volunteered because they had tinnitus while the other group volunteered because they had a hearing impairment. This difference in absolute thresholds between the groups presents a difficulty when interpreting the better performance on the compression and frequency selectivity measures in the tinnitus group. It could be that the worse indicators in the notinnitus group reflected nothing more than higher thresholds. However, the differences persisted after removing tinnitus participants with lower thresholds to equate the groups in terms of overall threshold (and, incidentally, age).

The compression measures were clearly different between the tinnitus and no-tinnitus samples at all frequencies. Moreover, reducing the tinnitus group did not have much effect on the average TMC slope. The TMC slope may therefore be a rather robust indicator of tinnitus. The pattern of frequency selectivity differences between the two groups was more complex; the no-tinnitus sample showed lowfrequency selectivity at all frequencies but the tinnitus group showed more frequency selectivity in the midfrequency range. These results can be explained with reference to the IFMC depth measurements made with the good-hearing sample at higher probe levels (Fig. 4), showing reduced depth at higher probe levels particularly at higher probe frequencies. It could be argued that the tinnitus group data were similar to what would be expected from the normal group if they had been tested at higher probe levels.

The impetus for this study was the availability of a new rapid testing procedure for generating auditory profiles and the realisation that it offered an opportunity to address the issue of whether tinnitus associated with hearing impairment might also be associated with a particular pattern of impairment. The results indicate that tinnitus is associated with better low-frequency thresholds, worse high-frequency thresholds, greater frequency selectivity and less compression than a no-tinnitus control group matched for overall hearing loss and age. This supports the idea that tinnitus may be triggered at least initially by a specific pattern of peripheral deficits even if it is later maintained centrally.

These results are open to interpretation in terms of OHC function. Psychophysical estimates of compression (as measured by the TMC) and frequency selectivity (as measured by the IFMC) are commonly thought to reflect the nonlinear response of the inner ear and to indicate the integrity of OHC function (Evans 1975; Liberman and Dodds 1984; Hicks and
Bacon 1999; Nelson et al. 2001; Robles and Ruggero 2001; Lopez-Poveda et al. 2005). If this is the case, then the tinnitus group performance might reflect a useful amount of residual OHC function compared to the no-tinnitus group. It certainly makes it difficult to attribute tinnitus to OHC dysfunction. Rather it is more likely that an explanation is to be found with IHC dysfunction or reduced innervation of the auditory nerve (Kujawa and Liberman 2009).

It is appreciated that these results appear to be inconsistent with numerous other studies that have inferred OHC rather than IHC dysfunction in association with tinnitus (see for example, Dauman and Cazals 1989; Mitchell and Creedon 1995; Zhou et al. 2011). There may be many reasons for this discrepancy but it should be noted that the current study used (1) psychophysical measurements made over a comprehensive range of frequencies, (2) a sample size larger than many other studies and (3) a threshold-matched and age-matched control group. While it is true that the tinnitus group had worse frequency selectivity and shallower compression functions than the good-hearing reference group, the difference might be explained in large part by the different levels used in the evaluation. Given the age of the tinnitus group, it is likely that some of the tinnitus group do have OHC dysfunction, but the evidence suggests that this is relatively minor compared to the no-tinnitus group. Nevertheless, it is not possible to rule out $\mathrm{OHC}$ involvement entirely.

The difficulties of interpreting the present results relative to other studies in terms of underlying pathology should not obscure the main result of the present study that a sample of hearing-impaired individuals reporting mild but persistent tinnitus showed a distinctive pattern of psychophysically assessed hearing when compared to a thresholdmatched and age-matched control group.

\section{ACKNOWLEDGMENTS}

The authors would like to thank M. Panda and N. Clark for insights and comments on the research. Research was funded by Action on Hearing Loss and the Overseas Research Student Awards Scheme.

\section{Conflict of interest}

The authors declare that they have no conflict of interest in the research.

\section{REFERENCES}

Bauer CA, Brozoski TJ, Myers K (2007) Primary afferent dendrite degeneration as a cause of tinnitus. J Neurosci Res 85:1489-1498 
Dauman R, Cazals Y (1989) Auditory frequency selectivity and tinnitus. Arch Otorhinolaryngol 246:252-255

Davis A, El Refaie A (2000) Epidemiology of tinnitus. In: Tyler RS (ed) Tinnitus handbook. Singular, San Diego

Dehmel S, Pradhan S, Koehler S, Bledsoe S, Shore S (2012) Noise overexposure alters long-term somatosensory auditory processing in the dorsal cochlear nucleus-possible basis for tinnitusrelated hyperactivity? J Neurosci 32:1660-1671

Evans EF (1975) The sharpening of cochlear frequency selectivity in the normal and abnormal cochlea. Audiology 14:419-442

HAwKINS JE JR (1973) Comparative otopathology: aging, noise, and ototoxic drugs. Adv Otorhinolaryngol 20:125-141

Hicks ML, BACON SP (1999) Effects of aspirin on psychophysical measures of frequency selectivity, two-tone suppression, and growth of masking. J Acoust Soc Am 106:1436-1451

Kaltenbach JA, Zhang J, Finlayson P (2005) Tinnitus as a plastic phenomenon and its possible neural underpinnings in the dorsal cochlear nucleus. Hear Res 206:200-226

Kujawa SG, Liberman MC (2009) Adding insult to injury: cochlear nerve degeneration after "temporary" noise-induced hearing loss. J Neurosci 29:14077-14085

Lecluyse W, Meddis R (2009) A simple single-interval adaptive procedure for estimating thresholds in normal and impaired listeners. J Acoust Soc Am 126:2570-2579

Liberman MC, Dodds LW (1984) Single-neuron labeling and chronic cochlear pathology. III. Stereocilia damage and alterations of threshold tuning curves. Hear Res 16:55-74

Lockwood AH, SAlvi R, Burkard RF (2002) Tinnitus. N Engl J Med 347:904-910
Lopez-Poveda EA, Plack CJ, Meddis R (2003) Cochlear nonlinearity between 500 and $8000 \mathrm{~Hz}$ in listeners with normal hearing. J Acoust Soc Am 113:951-960

Lopez-Poveda EA, Plack CJ, Meddis R, Blanco JL (2005) Cochlear compression in listeners with moderate sensorineural hearing loss. Hear Res 205:172-183

Meddis R, Lecluyse W, Tan CM, Panda MR, Ferry RT (2010) Beyond the audiogram: identifying and modelling patterns of hearing deficits. In: Lopez-Poveda EA, Palmer AR, Meddis R (eds) The neurophysiological bases of auditory perception. Springer, New York

Mitchell CR, Creedon TA (1995) Psychophysical tuning curves in subjects with tinnitus suggest outer hair cell lesions. Otolaryngol Head Neck Surg 113:223-233

Nelson DA, Schroder AC, Wojtczak M (2001) A new procedure for measuring peripheral compression in normal-hearing and hearing-impaired listeners. J Acoust Soc Am 110:2045-2064

Newman CW, Jacobson GP, Spitzer JB (1996) Development of the Tinnitus Handicap Inventory. Arch Otolaryngol Head Neck Surg 122:143-148

Nordmann AS, Bohne BA, Harding GW (2000) Histopathological differences between temporary and permanent threshold shift Hear Res 139:13-30

Robles L, Ruggero MA (2001) Mechanics of the mammalian cochlea. Physiol Rev 81:1305-1352

SASAKI CT, Babitz L, Kauer JS (1981) Tinnitus: development of a neurophysiologic correlate. Laryngoscope 91:2018-2024

Zhou X, Henin S, Long GR, Parra LC (2011) Impaired cochlear function correlates with the presence of tinnitus and its estimated spectral profile. Hear Res 277:107-116 